\title{
Expression of Neurotrophic Factors, Tight Junction Proteins, and Cytokines According to the Irritable Bowel Syndrome Subtype and Sex
}

\author{
Ju Yup Lee, ${ }^{1,2}$ Nayoung Kim, ${ }^{1,3 *}$ Ji Hyun Park, ${ }^{3}$ Ryoung Hee Nam, ${ }^{1}$ Sun Min Lee, ${ }^{1}$ Chin-Hee Song, ${ }^{1}$ Geun Kim, ${ }^{1}$ Hee Young Na, ${ }^{4}$ \\ Yoon Jin Choi, ${ }^{1}$ Jin Joo Kim, ${ }^{5}$ and Dong Ho Lee ${ }^{1,3}$ \\ Departments of ${ }^{1}$ Internal Medicine, ${ }^{4}$ Pathology, Seoul National University Bundang Hospital, Seoungnam, Gyeonggi-do, Korea; ${ }^{2}$ Department \\ of Internal Medicine, Keimyung University School of Medicine, Daegu, Korea; ${ }^{3}$ Department of Internal Medicine and Liver Research Institute, \\ Seoul National University College of Medicine, Seoul, Korea; and ${ }^{5}$ Department of Internal Medicine, Gyeongsang National University Changwon \\ Hospital, Changwon, Gyeongsangnam-do, Korea
}

\section{Background/Aims}

Emerging evidence shows that the mechanism of irritable bowel syndrome (IBS) is associated with neurotrophic factors and tight junction proteins (TJPS). It is known that there are sex differences in the pathophysiology of IBS. The aim of the present study is to determine expression levels of neurotrophic factors, TJPs, and cytokines according to IBS subtype and sex.

\section{Methods}

From 59 IBS (33 IBS-constipation, 21 IBS-diarrhea, and 5 IBS-mixed) and 36 control patients, colonic mucosa mRNA expression levels of transient receptor potential vanilloid-1 (TRPV1), nerve growth factor (NGF), glial cell-derived neurotrophic factor (GDNF), and various TJPs were assessed by real-time polymerase chain reaction. Western blot was performed to determine levels of zonular occludens-1 (ZO-1). Serum levels of cytokines were measured by enzyme-linked immunosorbent assay.

\section{Results}

TRPV1, GDNF, and NGF mRNA levels were significantly increased in those with IBS-constipation compared to those in controls (all $P$ $<0.05$ ). However, they showed no significant difference between those with IBS-diarrhea and controls. Expression level of TRPV1 correlated with that of GDNF $(r=0.741, P<0.001)$ and NGF $(r=0.935, P<0.001)$. ZO-1 RNA expression levels were lower $(P=$ 0.021 ) in female IBS-diarrhea than those in controls, although they showed no significant differences between male IBS-diarrhea and controls. Serum IL-1 $\beta$ levels in female IBS were significantly higher than those of male IBS, especially in IBS-constipation $(P<0.001)$.

\section{Conclusion}

Our results suggest that neurotrophic factors and IL-1 $\beta$ are closely related to IBS-constipation and that decrease of ZO-1 is an important factor in female with IBS-diarrhea.

(J Neurogastroenterol Motil 2020;26:106-116)

Key Words

Cytokines; Irritable bowel syndrome; Sex; Subtype; Tight junctions

Received: May 8, 2019 Revised: August 26, 2019 Accepted: September 27, 2019

(.) This is an Open Access article distributed under the terms of the Creative Commons Attribution Non-Commercial License (http://creativecommons. org/licenses/by-nc/4.0) which permits unrestricted non-commercial use, distribution, and reproduction in any medium, provided the original work is properly cited.

*Correspondence: Nayoung Kim, MD, PhD Department of Internal Medicine, Seoul National University Bundang Hospital, 82, Gumi-ro 173beon-gil, Bundang-gu, Seongnam, Gyeonggi-do 13620, Korea

Tel: +82-31-787-7008, Fax: +82-31-787-4051, E-mail: nayoungkim49@empas.com 


\section{Introduction}

Irritable bowel syndrome (IBS) is a common functional bowel disorder of unclear etiology characterized by recurrent abdominal pain or discomfort. ${ }^{1}$ The prevalence of IBS by the Rome III criteria in the general population is $1.1 \%$ to $29.2 \%$ worldwide. ${ }^{2}$ It is approximately $10.0 \%$ in Korea. ${ }^{2}$ IBS occurrence is known to be higher in females than that in males, with odd ratio of $1.67 .{ }^{3,4}$ In the literature, there are several reports regarding sex and gender differences in terms of IBS subtypes and response to IBS treatment. ${ }^{4-6}$ For instance, IBS-constipation (IBS-C) is frequent in females whereas IBS-diarrhea (IBS-D) is predominant in males. ${ }^{4-6}$ Despite there are a few reports regarding sex and gender difference of IBS in animal studies, ${ }^{7,8}$ causes of vulnerability of females to IBS remain unclear. ${ }^{5,9}$ So far, most IBS studies have focused on IBS-D or post-infectious IBS (PI-IBS) that develops into IBS-D or on mixed type IBS (IBS-M). Importantly, only a few papers have illustrated research regarding IBS-C pathophysiology. ${ }^{10}$

The possible pathophysiology of IBS is multifactorial., ${ }^{6,11}$ Among them, emerging data highlight that neurotrophic factors and altered intestinal permeability might contribute to IBS and related symptoms. Increased attention has been also given to the neural-immune-endocrine network mechanism in IBS-D. Nerve growth factor (NGF) and glial cell-derived neurotrophic factor (GDNF) are closely related to the pathogenesis of IBS. ${ }^{12}$ In addition, transient receptor potential vanilloid-1 (TRPV1) is believed to be an important integrator of the transmission and modulation of pain with NGF or GDNF. ${ }^{13}$

A previous study has demonstrated that tight junction proteins (TJPs) such as zonula occludens-1 (ZO-1) and occludin (OCLN) expression are lower in IBS patients than those in controls. ${ }^{14}$ In subgroup analyses, OCLN and claudin-1 (CLDN1) expression levels are decreased in IBS-D patients, but not in IBS-C or IBS-M patients. ${ }^{14}$ However, a study that compares the degree of expression of TJPs depending on IBS subtype and sex has not been reported yet. Various inflammatory cytokines are known to be involved in the pathophysiology of IBS. ${ }^{15,16}$ Previous studies have shown that serum levels of pro-inflammatory cytokines such as IL-1, IL-8, and TNF- $\alpha$ are increased in IBS patients. ${ }^{17}$ Furthermore, there are differences in types of cytokines recruited according to their origin, colon mucosa, or blood of IBS patients. Importantly, only a few studies regarding blood cytokines in IBS patients have reflected systemic effects.

With such background, we hypothesized that the mechanisms underlying IBS could be different depending on IBS subtype and sex. Thus, the aim of this study is to evaluate expression levels of neurotrophic factors, TJPs, and cytokines in IBS patients and clarify sex differences in the pathophysiology of IBS.

\section{Materials and Methods}

\section{Subjects}

Subjects were prospectively enrolled at the Department of Gastroenterology of Seoul National University Bundang Hospital between September 2013 and September 2017. Subjects were assigned into IBS or control group according to the Rome III criteria. The control group was defined as subjects without gastrointestinal symptoms or colonoscopic lesions. All subjects were from Korea and the control group consisted of subjects who underwent colonoscopy for the purpose of health checkup. They received colonoscopies and questionnaires about IBS symptoms under the supervision of a well-trained interviewer. IBS patients were categorized into IBS-D, IBS-C, IBS-M, and unsubtyped (IBS-U) on the basis of the Rome III criteria. We excluded PI-IBS patients. This study was approved by the Institutional Review Board of SNUBH (Approval No. B-1305/202-003). Written informed consent was obtained from all subjects. This study protocol has been registered at ClinicalTrials.gov (NCT03675100).

\section{Symptom Assessment Questionnaire}

A validated Rome III criteria-based bowel disease questionnaire was translated from the original Bowel Disease Questionnaire into Korean (Korean Bowel Disease Questionnaire) and used to investigate patient's bowel symptoms. ${ }^{18,19}$ Patients were asked to rate frequency and severity of symptoms experienced over the last 3 months prior to the study. The severity of abdominal pain was scored from 1 to 5 according to its impact on patients' daily activities: 1 , mild; 2 , relevant; 3 , moderate; 4 , severe; and 5 , extremely severe. The frequency of abdominal pain was graded from 0 to 6 according to the following scale: 0 , absent; 1 , less than once a month; 2 , 1 day/month; 3, 2-3 days/month; 4, once a week; 5, 2-3 days/week; and 6, daily.

\section{Colonoscopic Biopsy}

During colonoscopic examination, 2 biopsies were carefully taken from normal looking sigmoid colon mucosa of each subject using standard biopsy forceps. Samples were processed for mRNA expression analysis of neurotrophic factors and TJPs or Western 
blot analysis for detecting ZO-1. In addition, if subjects agreed to provide a biopsy for immunohistochemical (IHC) analysis of ZO1 , another biopsy specimen was collected using the same method.

\section{Real-time Quantitative Polymerase Chain Reaction}

In order to stabilize and protect RNA from degradation, biopsy specimens were stored in RNAlater Solution (Ambion, Austin, TX, USA) at $4^{\circ} \mathrm{C}$ after endoscopy. Total RNAs were extracted from these colonic mucosal biopsy specimens using TRIzol reagent (Invitrogen, Carlsbad, CA, USA) as recommended by the manufacturer. Extracted RNA was purified using RNeasy mini kits (Qiagen, Valencia, CA, USA). Synthesis of cDNA was performed using 1 $\mu \mathrm{g}$ of total RNA with a High Capacity cDNA kit (Applied Biosystems, Foster City, CA, USA) according to the manufacturer's instructions. Thermal cycling parameters for reverse transcription were: 10 minutes at $25^{\circ} \mathrm{C}, 120$ minutes at $37^{\circ} \mathrm{C}$, and 5 minutes at $85^{\circ} \mathrm{C}$. Real-time quantitative polymerase chain reaction was performed in triplicates using a StepOnePlus Real-time PCR (Applied Biosystems) with SYBR Premix Ex TaqTM (Takara Bio, Shiga, Japan) according to manufacturers' instructions and protocols. Primers used in this study followed those published previously. ${ }^{20,21}$ Thermal cycling was then performed as follows: initial denaturation at $95^{\circ} \mathrm{C}$ for 10 seconds followed by 40 cycles of $95^{\circ} \mathrm{C}$ for 5 seconds and $60-65^{\circ} \mathrm{C}$ for 33 seconds. Expression levels of mRNAs for target genes were compared with endogenous control $\beta$-actin using the $2^{-\Delta \mathrm{Ct}}$ method.

\section{Western Blot Analysis and Immunohistochemical Analysis of Zonula Occludens-1}

Total protein was extracted from colon tissue using radioimmunoprecipitation assay buffer (Cell Signaling Technology, Beverly, MA, USA) containing protease and phosphatase inhibitors. After electrophoresis, proteins were transferred to polyvinylidene difluoride membranes. Rabbit polyclonal antibodies (Santa Cruz Biotechnology, Santa Cruz, CA, USA) against ZO-1 and $\beta$-actin were used as primary antibodies. An imaging analyzer was used to measure band densities. For IHC, ZO-1 tissue sections were treated with $3 \%$ hydrogen peroxide and nonspecific binding sites were blocked. Sections were then incubated with anti-ZO-1 antibodies (61-7300; Thermo Fisher Scientific, Inc, Waltham, MA, USA). An automatic immunostainer (BenchMark XT; Ventana Medical Systems, Inc, Tucson, AZ, USA) and an UltraView Universal DAB detection kit (Ventana Medical Systems) were used for immunostaining. ${ }^{22}$ Expression percentage was assessed as a ratio between the number of cells stained with ZO-1 divided by the total

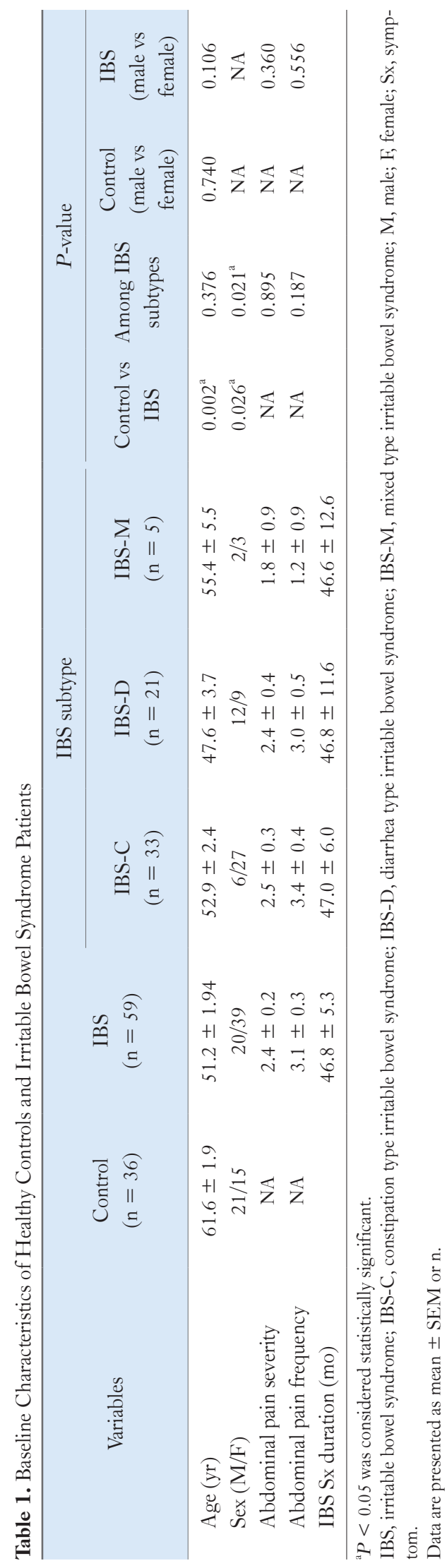


number of cells counted in a section. ZO-1 expression was quantified using Image J $1.50 \mathrm{i}$ software. Reported values are the average of different images from different subjects within each group.

\section{Measurement of Serum Cytokines}

Collected serum samples were immediately frozen at $-20^{\circ} \mathrm{C}$ and then stored at $-80^{\circ} \mathrm{C}$ until further laboratory analyses were carried out. Based on existing literature describing alterations between IBS patients and healthy controls, serum levels of TNF- $\alpha$, IL-1 $\beta$, and IL-8 were measured using enzyme-linked immunosorbent assay kits (R\&D Systems, Minneapolis, MN, USA).

\section{Statistical Methods}

SPSS for Windows (version 18.0; SPSS, Chicago, IL, USA) was used for all statistical analyses. Data are expressed as mean \pm standard error of the mean. Continuous variables were analyzed using Kruskal-Wallis test or Mann-Whitney test. Mann-Whitney test was done as a post-hoc test. Fisher's exact test was used for analysis of categorical variables. Spearman rank correlation analysis was performed to determine correlation between 2 variables. All results were considered statistically significant when $P$-values were less than 0.05 .

\section{Results}

\section{General Characteristics}

Baseline characteristics of 59 IBS patients and 36 control sub-

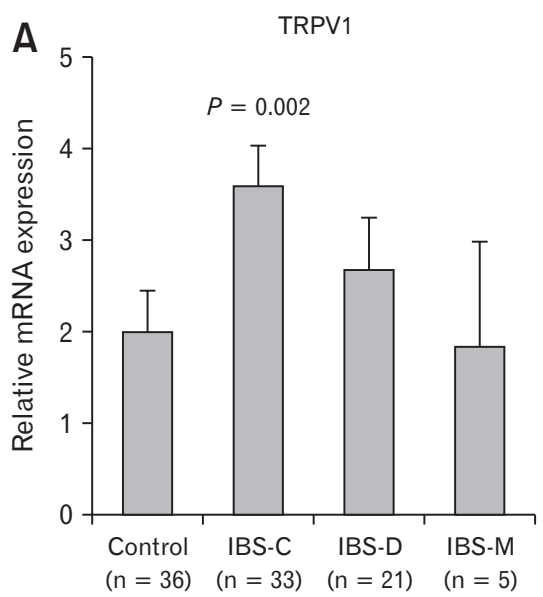

C

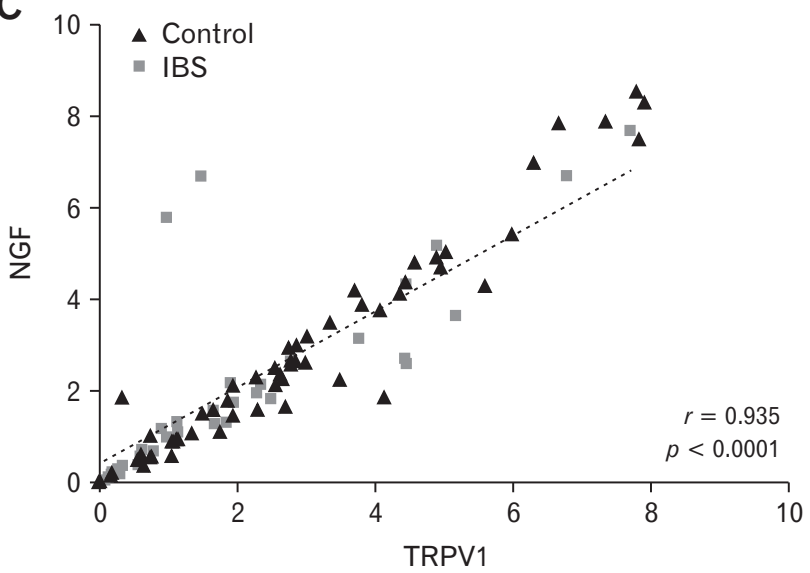

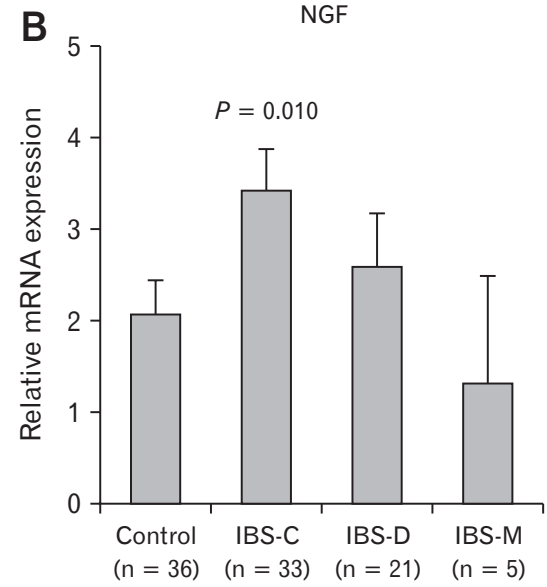

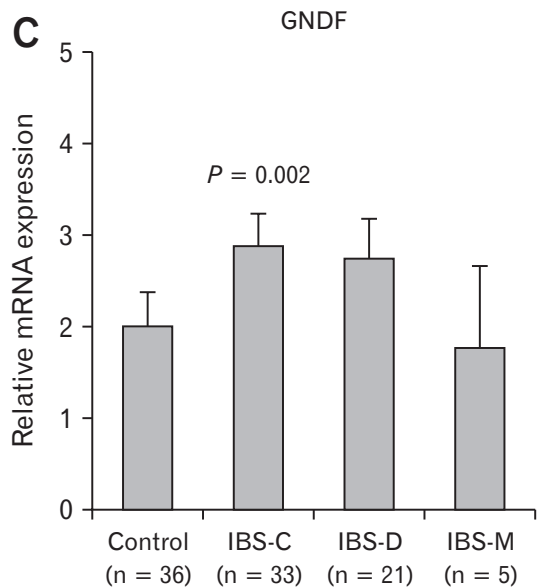

Figure 1. Transient receptor potential vanilloid-1 (TRPV1), nerve growth factor (NGF), and glial derived neurotrophic factor (GDNF) mRNA expression levels in the colonic mucosa of irritable bowel syndrome (IBS) patients. (A-C) Significantly higher expression levels of TRPV1, NGF, and GDNF were observed in constipation type IBS (IBS-C) patients compared to controls. (D, E) There was a strong positive relationship between TRPV1 and NGF or GDNF $(r=0.935, P<0.0001$ for NGF; $r=0.741, P<0.0001$ for GDNF). IBS-D, diarrhea type irritable bowel syndrome; IBS-M, mixed type irritable bowel syndrome. 
jects are shown in Table 1. Compared to controls, IBS patients were younger $(P=0.002)$ and female patients were predominant in IBS $(P=0.026)$. Abdominal pain severity and frequency were similar among the 3 IBS subtypes. These parameters showed no sexspecific differences in IBS.

\section{Comparing Transient Receptor Potential Vanilloid-1, Nerve Growth Factor, and Glial Cell-derived Neurotrophic Factor Expression Levels Between Irritable Bowel Syndrome and Control}

TRPV1 mRNA levels in colonic mucosa were significantly increased in IBS patients than those in controls. In subgroup analysis, only IBS-C group showed significant higher levels of TRPV1
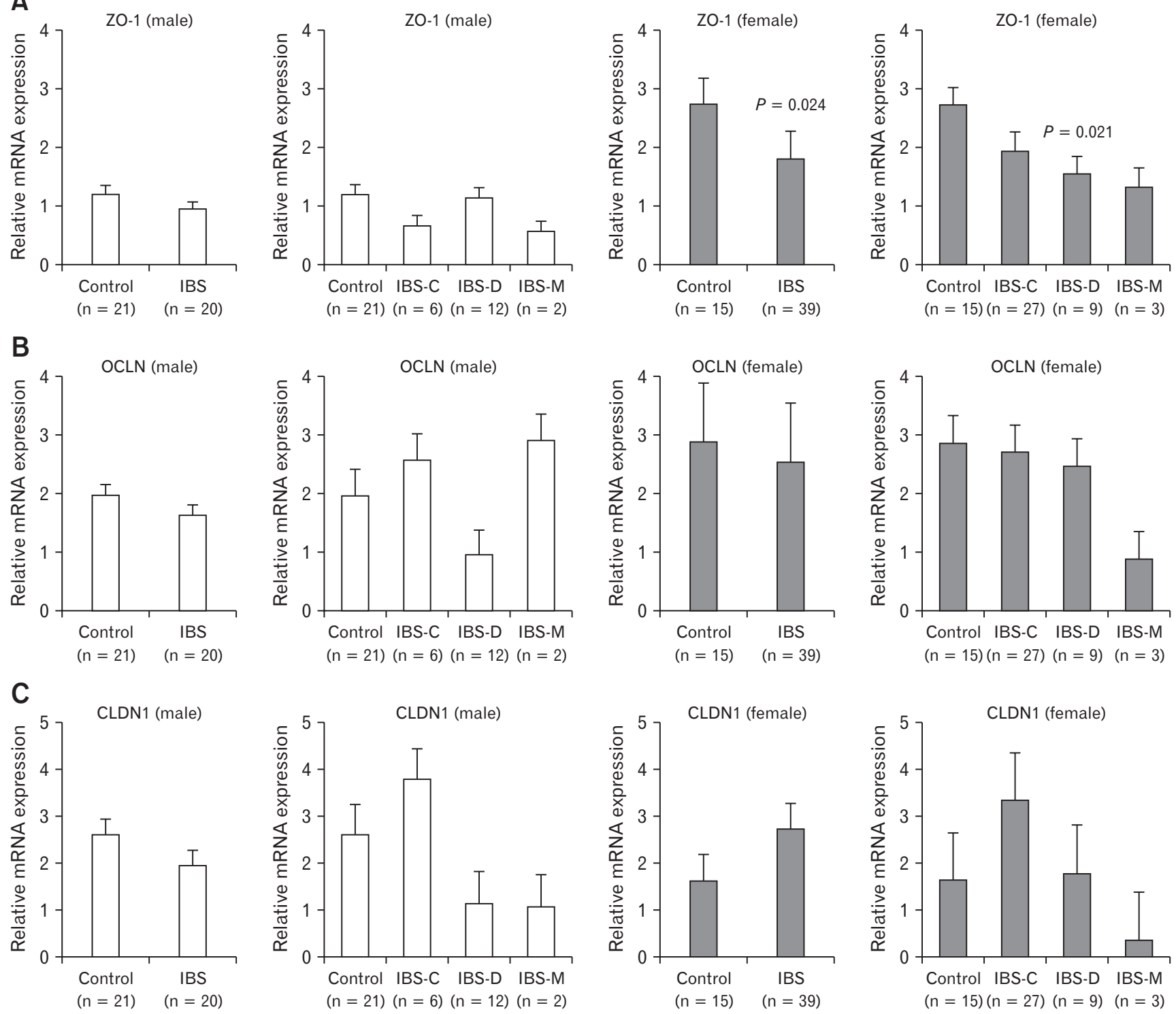

Figure 2. Zonula occludens-1 (ZO-1), occludin (OCLN), and claudin-1 (CLDN1) mRNA expression levels in the colonic mucosa of IBS patients according to IBS subtype and sex. (A) Female irritable bowel syndrome (IBS) patients showed significantly lower ZO-1 mRNA expression levels, especially in diarrhea type IBS (IBS-D) and mixed type IBS (IBS-M) than control females $(P=0.021)$. (B, C) OCLN and CLDN1 mRNA expression levels were similar among subtypes and sexes. IBS-C, constipation type irritable bowel syndrome. 
than the control group $(P=0.002)$ (Fig. 1A). NGF and GDNF $m R N A$ levels were also significantly increased in IBS patients than those in controls, especially those in IBS-C $(P=0.010$ and $P=$ 0.002 , respectively) (Fig. $1 \mathrm{~B}$ and $1 \mathrm{C}$ ). There were no significant sex differences in TRPV1, NGF, or GDNF expression. When the relationship between TRPV1 and NGF or GDNF expression was

Table 2. Zonular Occludens-1, Occludin, and Claudin-1 Expression in the Colonic Mucosa According to the Severity of Abdominal Pain in Irritable Bowel Syndrome Patients

\begin{tabular}{llccc}
\hline \multirow{7}{*}{ IBS } & & $\begin{array}{c}\text { Pain severity } \\
1-3\end{array}$ & $\begin{array}{c}\text { Pain severity } \\
4-5\end{array}$ & $\begin{array}{c}P \text {-value } \\
\end{array}$ \\
& ZO-1 & $0.77(1.76)$ & $0.45(0.75)$ & 0.075 \\
& OCLN & $1.78(1.59)$ & $0.54(1.16)$ & $0.005^{\text {a }}$ \\
& CLDN1 & $2.23(3.80)$ & $0.29(1.71)$ & $0.036^{\text {a }}$ \\
IBS-C & ZO-1 & $1.04(1.85)$ & $0.69(0.99)$ & 0.578 \\
& OCLN & $1.78(1.51)$ & $3.31(2.52)$ & 0.284 \\
& CLDN1 & $3.05(3.96)$ & $7.48(0.86)$ & $0.032^{\text {a }}$ \\
IBS-D & ZO-1 & $0.69(1.20)$ & $0.51(0.45)$ & 0.601 \\
& OCLN & $1.54(2.27)$ & $0.51(0.60)$ & 0.067 \\
& CLDN1 & $0.88(3.15)$ & $0.29(1.25)$ & 0.149 \\
IBS-M & ZO-1 & $2.25(3.32)$ & $0.05(0.04)$ & 0.333 \\
& OCLN & $2.53(1.28)$ & $0.49(0.85)$ & 0.200 \\
& CLDN1 & $1.00(0.40)$ & $0.20(0.19)$ & 0.200 \\
\hline
\end{tabular}

${ }^{a} P<0.05$ was considered statistically significant.

IBS, irritable bowel syndrome; IBS-C, constipation type irritable bowel syndrome; IBS-D, diarrhea type irritable bowel syndrome; IBS-M, mixed type irritable bowel syndrome; ZO-1, zonula occludens-1; OCLN, occludin; CLDN1, claudin-1. analyzed, a strong positive correlation was observed $(r=0.935, P<$ 0.0001 in NGF; $r=0.741, P<0.0001$ in GDNF) (Fig. $1 \mathrm{D}$ and $1 \mathrm{E})$.

Table 3. Multivariate Analysis of Sex, Irritable Bowel Syndrome Types, Neurotrophic Factors, and Tight Junction Proteins With Abdominal Pain Severity

\begin{tabular}{lcc}
\hline \multicolumn{1}{c}{ Variable } & Odds ratio $(95 \% \mathrm{CI})$ & $P$-value \\
\hline Female sex & $0.106(0.006-1.866)$ & 0.125 \\
Age & $0.945(0.872-1.024)$ & 0.170 \\
IBS-C & 1.000 (reference) & \\
IBS-D & $1.805(0.095-34.350)$ & 0.695 \\
IBS-M & $33.193(0.285-3872.180)$ & 0.149 \\
TRPV1 mRNA & $1.673(0.291-9.622)$ & 0.564 \\
NGF mRNA & $1.490(0.250-8.865)$ & 0.661 \\
GDNF mRNA & $0.495(0.201-1.218)$ & 0.126 \\
ZO-1 mRNA & $0.407(0.081-2.044)$ & 0.275 \\
Occludin mRNA & $0.167(0.017-1.650)$ & 0.126 \\
Claudin-1 mRNA & $0.987(0.424-2.294)$ & 0.975 \\
\hline
\end{tabular}

IBS-C, constipation type irritable bowel syndrome; IBS-D, diarrhea type irritable bowel syndrome; IBS-M, mixed type irritable bowel syndrome; TRPV1, transient receptor potential vanilloid-1; NGF, nerve growth factor; GDNF, glial derived neurotrophic factor; ZO-1, zonula occludens-1.
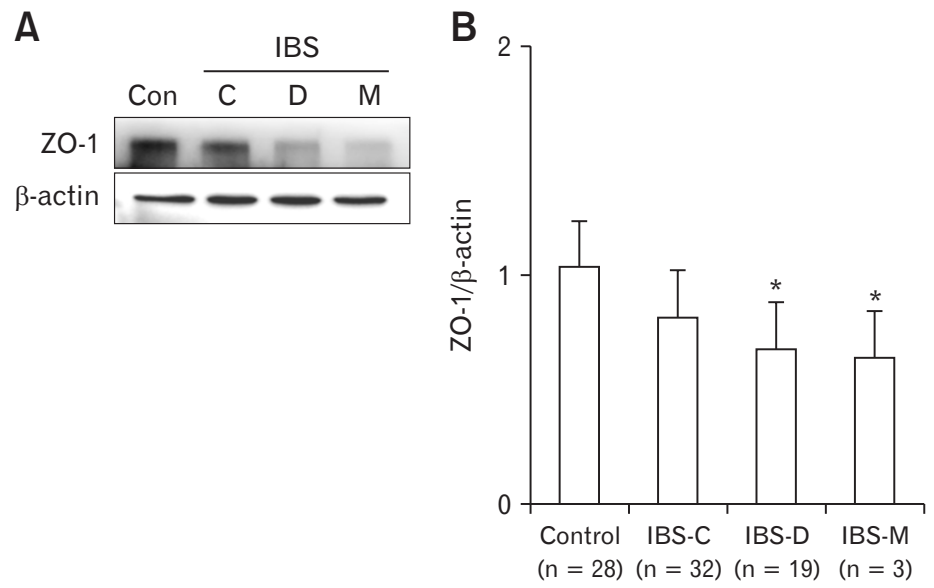

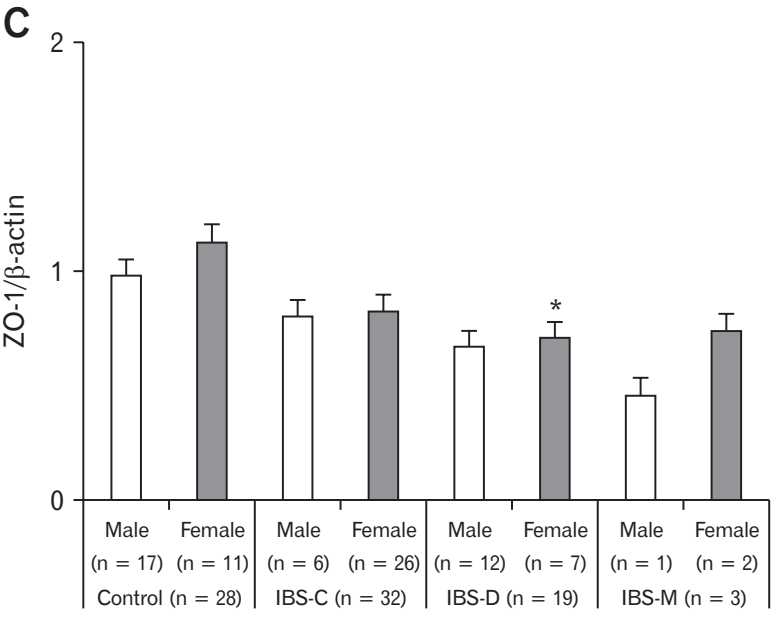

Figure 3. Western blot analysis of zonula occludens-1 (ZO-1) mRNA expression level in each irritable bowel syndrome (IBS) subtype and sex. (A, B) ZO-1 mRNA expression levels in diarrhea type IBS (IBS-D) and mixed type IBS (IBS-M) patients were significantly lower than those of the control group. (C) Sex difference of ZO-1 mRNA expression in IBS-D compared with control ( $\left.{ }^{\star} P<0.05\right)$. Con, control; C, constipation type; D, diarrhea type; M, mixed type; IBS-C, constipation type irritable bowel syndrome. 


\section{Comparison of Tight Junction Protein-related Genes' Expression Levels and Association Between Expression of Tight Junction Proteins and Irritable Bowel Syndrome Symptoms}

Female IBS-D patients showed significantly lower ZO-1 mRNA levels than females in the control group $(2.74 \pm 0.67$ vs $1.55 \pm 0.80, P=0.021$ ) (Fig. 2A). However, no differences in ZO-1 mRNA levels were observed in males. OCLN and CLDN1 mRNA expression levels were similar among the 3 IBS subtypes (Fig. 2B and 2C). While ZO-1 mRNA expression was not sig- nificantly different according to the severity of abdominal pain, OCLN and CLDN1 expression levels were significantly lower in IBS patients with a pain severity score of 4 or 5 . In IBS-C patients, OCLN and CLDN1 expression levels were increased according to abdominal pain severity. In addition, CLDN1 expression was significantly higher in abdominal pain severity 4-5 group $(P=$ 0.032). In IBS-D patients, expression levels of ZO-1, OCLN, and CLDN1 were generally decreased as absolute values with increasing abdominal pain severity, although these decreases were not statistically significant (Table 2). Multivariate analysis was performed to identify factors associated with IBS symptoms severity. However,
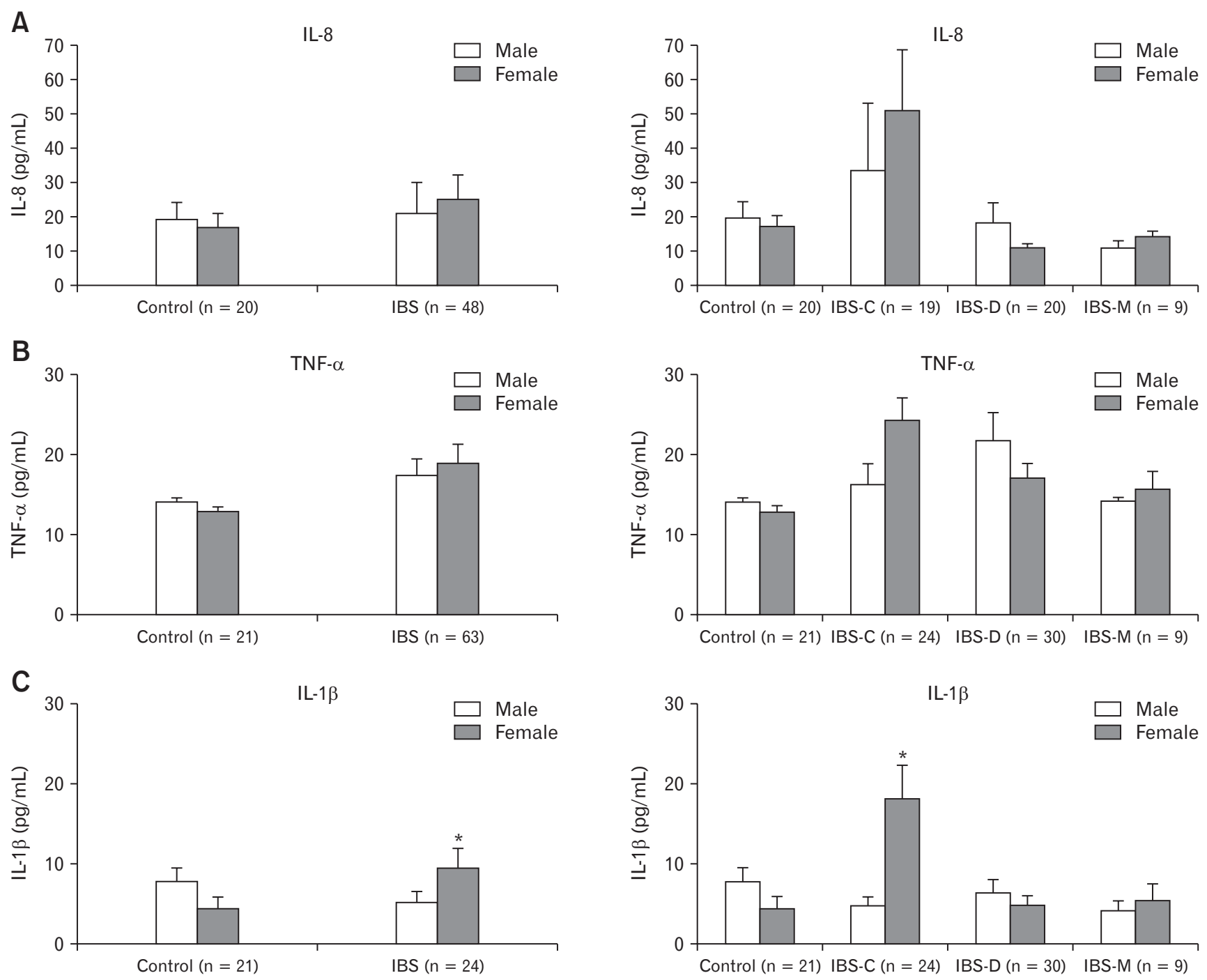

Figure 4. Serum levels of (A) interleukin-8 (IL-8), (B) tumor necrosis factor- $\alpha$ (TNF- $\alpha$ ), and (C) IL-1 $\beta$ cytokines in irritable bowel syndrome (IBS) patients and controls according to IBS subtype and sex. (C) Serum IL-1 $\beta$ level was significantly higher in female IBS patients than that in male IBS patients, especially in constipation type IBS (IBS-C) $\left({ }^{\star} P<0.001\right)$. IBS-D, diarrhea type irritable bowel syndrome; IBS-M, mixed type irritable bowel syndrome. 
Table 4. Summary of All Significant Variables Found in Irritable Bowel Syndrome Patients

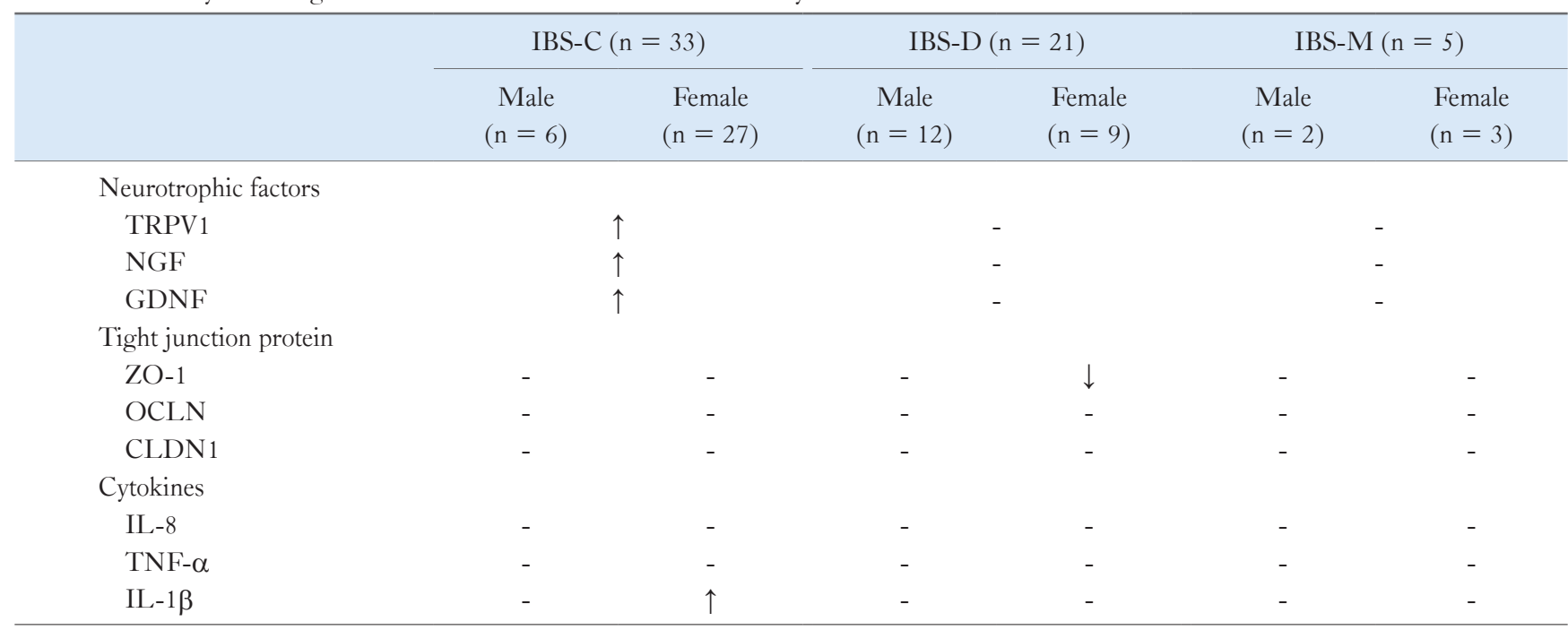

IBS-C, constipation type irritable bowel syndrome; IBS-D, diarrhea type irritable bowel syndrome; IBS-M, mixed type irritable bowel syndrome; TRPV1, transient receptor potential vanilloid-1; NGF, nerve growth factor; GDNF, glial derived neurotrophic factor; ZO-1, zonula occludens-1; OCLN, occludin; CLDN1, claudin-1; $\uparrow$, significantly increased; $\downarrow$, significantly decreased; -, not significant.

no factor was significantly related to the severity of abdominal pain (Table 3).

\section{Western Blot Analysis of Zonula Occludens-1}

In Western blot analysis, relative expression levels of ZO-1 in IBS-D and IBS-M were significantly lower than those in controls. In addition, relative expression level of ZO-1 showed sex difference in IBS-D in comparison with controls (Fig. 3).

\section{Serum Levels of Interleukin-8, Tumor Necrosis Factor- $\alpha$, and Interluekin- $1 \beta$ Cytokines in Irritable Bowel Syndrome and Control Groups According to Sex}

Serum levels of IL-8, TNF- $\alpha$, and IL- $1 \beta$ cytokines were increased in IBS patients than those in controls. In addition, serum levels of IL-1 $\beta$ were significantly increased in female IBS patients than those in males. In subgroup analysis according to IBS subtype, IL-8, TNF- $\alpha$, and IL-1 $\beta$ levels were increased in female IBS-C patients than in males, although IL- 8 and TNF- $\alpha$ levels were not significantly higher in female IBS-C patients. However, levels of IL-1 $\beta$ in female IBS-C patients were significantly increased compared to those in male IBS-C patients $(P<0.001)$ (Fig. 4).

\section{Discussion}

Recently, neural-immune-endocrine network including nerve growth factors has attracted increasing attention not only in IBS, ${ }^{11}$ but also in esophageal ${ }^{20,23,24}$ and gastric pathophysiology. ${ }^{25}$ In the esophagus, TRPV1 receptors and neurotropic factors have been reported to play an important role in the inflammatory process. ${ }^{23,24}$ Our team has also demonstrated that TRPV1, GDNF, and NGF are up-regulated in reflux esophagitis. ${ }^{20}$ Furthermore, functional dyspepsia group has higher expressions of TRPV1, GDNF, NGF mRNA, and TRPV1 staining than the control group. ${ }^{26}$ In the present study, TRPV1, NGF, and GDNF expression levels were significantly higher in IBS-C patients regardless of sex. In addition, a strong correlation was observed among these neurotrophic factors (Table 4). NGF promotes growth of neurons and tissues differentiation together with changes in plasticity of nerve fibers in case of acute chronic stress or tissue damage. NGF known to play a proalgesic role during inflammation is upregulated in colonic mucosa of IBS patients. ${ }^{27,28}$ GDNF belonging to the TGF- $\beta$ superfamily is overexpressed in IBS patients. ${ }^{29}$ It has been suggested that GDNF is involved in gastrointestinal sensory transduction together with TRPV $1 .{ }^{30}$ Recently, Xu et al ${ }^{27}$ have reported that NGF mRNA expression is significantly increased in colonic mucosal tissues of IBS$\mathrm{D}$ patients, interacting with mast cells and sensory nerve fibers in 38 Chinese IBS-D patients and 20 healthy controls. However, their study did not evaluate IBS-C patients. Furthermore, they did no distinguish between PI-IBS and non-PI-IBS.

In contrast, in our study that excluded PI-IBS patients, TRPV1, NGF, and GDNF expression levels were significantly 
increased only in IBS-C patients, but not in IBS-D ones. These differences may be due to different pathophysiologies of PI-IBS and non-PI-IBS. The exact cause for such differences is currently unclear. However, since serum IL- $1 \beta$ was closely related to IBS-C in females, but not in IBS-D in our study, neurotrophic factors and serum IL- $1 \beta$ might be associated with inflammation in IBS-C.

TJPs are multi-protein complexes that form a barrier which is a selectively permeable seal between adjacent epithelial cells. ${ }^{31}$ Modification of TJP molecules and paracellular permeability on barrier function are regulated by various extracellular stimuli. They are closely associated with maintenance of homeostasis. ${ }^{32} \mathrm{TJPs}$ consist of cytoplasmic proteins such as ZO-1 and transmembrane proteins such as occludin and claudin. ${ }^{33}$ Occludin and claudin-1 can interact with peripheral scaffolding protein ZO-1 which binds to actin cytoskeleton and microtubules. ${ }^{34}$ To date, there is a lack of research on the role of TJP in IBS. Piche et $\mathrm{al}^{35}$ have reported that increased paracellular permeability in IBS patients is associated with a decrease in ZO-1 mRNA level. Other studies have demonstrated a decrease in occludin expression from colonic mucosa in patients with IBS. ${ }^{15}$ It has been reported that decreased expression and altered cellular distribution of occludin and claudin-1 in IBS-D are correlated with the intensity of IBS symptoms. ${ }^{14}$ In our study, only ZO-1 expression was significantly decreased in IBS-D while differences in expression levels of OCLN and CLDN1 were minimal (Table 4). These differences might originate from the different roles between occludin/claudin and ZO-1. In particular, ZO-1 acts directly on actin-myosin. Their interaction might play an important role in IBS pathophysiology. ${ }^{31-33}$ In addition, the present study demonstrated that ZO-1 protein level was decreased based on Western blotting. The present study was important in that it compared IBSD and IBS-C while completely excluding PI-IBS, unlike other IBS-D studies that contained PI-IBS. The decrease in ZO-1 level was significant in female non-post infectious IBS-D. These results suggest that the mechanism of IBS in females might be different from that of males, although our results need to be confirmed with a larger sample in the future.

Increased colonic mucosal T lymphocytes ${ }^{36}$ and IL-1 $\beta^{37}$ have been reported in PI-IBS, with less consistency in non-PI-IBS. Elevated basal and stimulated cytokine levels in the blood have been reported in some IBS studies, but not in all IBS studies. Blood IL-6 and IL-8 levels were elevated in all IBS subgroups, with elevation being the most in IBS-C. ${ }^{38}$ IBS patients showed significantly higher baseline levels of TNF- $\alpha$, IL- $1 \beta$, and IL-6 than healthy controls. ${ }^{37}$ After analyzing IBS subgroups, all cytokine levels were significantly higher in IBS-D whereas IBS-C patients showed increased IL- $1 \beta$ levels than healthy controls. ${ }^{39}$ Chang et $\mathrm{al}^{40}$ have shown that mRNA expression level of anti-inflammatory cytokine IL-10 from sigmoid colon mucosa is lower in female IBS than controls. Interestingly, no difference was observed in males, thus advocating IL-10 as a potential biomarker for IBS. Cytokine levels in blood did not correlate with their respective expression levels in the sigmoid colon mucosa in IBS. ${ }^{40}$ There are a few studies on sex differences of various cytokines expressed in patients with IBS. In one animal study, colonic mucosal expression levels of IFN- $\gamma$, TNF- $\alpha$, IL-6, and IL-17 were higher in female IBS model rats, suggesting sex differences in cytokine expression. ${ }^{8}$ However, in another animal study, only IL-1 $\beta$ expression level was increased in the colonic mucosa of female IBS rats. ${ }^{7}$ It is important to note that research on the aforementioned aspects is still lacking. These conflicting results may be due to various reasons, such as differences in the method of quantification of cytokines and different intestinal mucosa samples used in each study. In our work, cytokines in the serum were significantly increased in IBS-C, especially IL- $1 \beta$ in female IBS-C (Table 4). This is contrary to the previously accepted concept of "more inflammation in IBS-D.” Peters et $\mathrm{al}^{10}$ have recently demonstrated normal colonic barrier and secretory function in female IBS-C. They have concluded that, although IBS-C and IBS-D are diagnosed and conceptualized using similar mechanistic framework, peripheral mechanisms of these subtypes are quite different.

Higher IL-1 $\beta$ levels in female IBS-C can be explained by activity of estradiol and inflammasome. $.^{25} \mathrm{IL}-1 \beta$ is expressed as an inactive pro-IL-1 $\beta$ moiety. It is activated by cleavage caspase-1. Activation of caspase- 1 is achieved by cytosolic multiprotein complexes called inflammasomes. A recent study has shown that estradiol can activate Nod-like receptor protein 3 inflammasome expression in female azoxymethane/dextran sulfate sodium mouse model. ${ }^{25} \mathrm{Al}-$ though further research is needed in the future, these findings suggest that serum IL- $1 \beta$ levels might be higher in female IBS patients than those in male IBS because of the effect of estradiol.

The present study has several limitations. First, the number of IBS patients enrolled in the present study was relatively small to perform adequate analysis for the IBS subtype. The number of controls was also small because it was not easy to obtain consent from subjects due to the invasiveness of colonic mucosal biopsy. Second, a bias could occur statistically in terms of the sex and IBS subtypes because ratios of sex and IBS subtypes did not match between controls and IBS patients. Third, we only focused on changes of braingut neurotrophic factor and intestinal microenvironment (TJP) according to IBS subtype. Thus, a complete analysis of brain-gutmicrobiome was not achieved. 
In conclusion, different expression levels of neurotrophic factors, neuropeptides, and cytokines were observed according to subtypes and sexes of IBS. Colonic mucosal TRPV1, NGF, and GDNF levels were higher in IBS-C while ZO-1 level was observed to be significantly lower in female IBS-D. Serum IL-1 $\beta$ levels were higher in female IBS-C. These findings suggest that the pathophysiology of IBS-C and IBS-D may be different and that IBS pathophysiology is dependent on the sex of an individual. These findings unveil novel opportunities for therapeutic targets in IBS. It might be possible to develop tailored therapy according to the sex of patient.

Acknowledgements: The authors wish to thank Sooin Choi and Sora Kang for technical assistance.

Financial support: This research was supported by Support Program for Women in Science, Engineering and Technology through the National Research Foundation of Korea (NRF) funded by the Ministry of Science and ICT (No. 2019H1C3A1032224).

\section{Conflicts of interest: None.}

Author contributions: Ju Yup Lee analyzed the data and drafted the manuscript, tables, and figures, and approved the final version to be published; Nayoung Kim designed the study, collected all samples, supervised the analyses, revised the draft, and approved the final version; Ji Hyun Park performed molecular studies; Ryoung Hee Nam and Sun Min Lee handled the samples of colon biopsy and blood; Chin-Hee Song performed Western blot study of ZO-1; Geun Kim reviewed the samples for ZO-1 IHC study; Hee Young $\mathrm{Na}$ analyzed the IHC study for ZO-1; and Yoon Jin Choi, Jin Joo Kim, and Dong Ho Lee provided criticism of the manuscript and approved the final version. All authors have read and approved the manuscript.

\section{References}

1. Longstreth GF, Thompson WG, Chey WD, Houghton LA, Mearin F, Spiller RC. Functional bowel disorders. Gastroenterology 2006;130:1480-1491.

2. Oshima T, Miwa H. Gastrointestinal mucosal barrier function and diseases. J Gastroenterol 2016;51:768-778.

3. Lovell RM, Ford AC. Global prevalence of and risk factors for irritable bowel syndrome: a meta-analysis. Clin Gastroenterol Hepatol 2012;10:712-721, e4.

4. Kim YS, Kim N. Sex and gender differences in irritable bowel syndrome. J Neurogastroenterol Motil 2018;24:544-558.
5. Houghton LA, Heitkemper M, Crowell M, et al. Age, gender and women's health and the patient. Gastroenterology 2016;150:1332-1343, e4.

6. Meleine M, Matricon J. Gender-related differences in irritable bowel syndrome: potential mechanisms of sex hormones. World J Gastroenterol 2014;20:6725-6743

7. Lee JY, Kim N, Kim YS, et al. Repeated water avoidance stress alters mucosal mast cell counts, interleukin-1beta levels with sex differences in the distal colon of Wistar rats. J Neurogastroenterol Motil 2016;22:694704.

8. Lee JY, Kim N, Nam RH, et al. Probiotics reduce repeated water avoidance stress-induced colonic microinflammation in Wistar rats in a sexspecific manner. PLoS One 2017;12:e0188992.

9. Chang L, Heitkemper MM. Gender differences in irritable bowel syndrome. Gastroenterology 2002;123:1686-1701.

10. Peters SA, Edogawa S, Sundt WJ, et al. Constipation-predominant irritable bowel syndrome females have normal colonic barrier and secretory function. Am J Gastroenterol 2017;112:913-923.

11. Barbara G, Cremon C, Carini G, et al. The immune system in irritable bowel syndrome. J Neurogastroenterol Motil 2011;17:349-359.

12. Yu YB, Zuo XL, Zhao QJ, et al. Brain-derived neurotrophic factor contributes to abdominal pain in irritable bowel syndrome. Gut 2012;61:685694.

13. Akbar A, Yiangou Y, Facer P, Walters JR, Anand P, Ghosh S. Increased capsaicin receptor TRPV1-expressing sensory fibres in irritable bowel syndrome and their correlation with abdominal pain. Gut 2008;57:923929.

14. Bertiaux-Vandaële N, Youmba SB, Belmonte L, et al. The expression and the cellular distribution of the tight junction proteins are altered in irritable bowel syndrome patients with differences according to the disease subtype. Am J Gastroenterol 2011;106:2165-2173.

15. Coëffier M, Gloro R, Boukhettala N, et al. Increased proteasomemediated degradation of occludin in irritable bowel syndrome. Am J Gastroenterol 2010;105:1181-1188.

16. Macsharry J, O'Mahony L, Fanning A, et al. Mucosal cytokine imbalance in irritable bowel syndrome. Scand J Gastroenterol 2008;43:14671476.

17. Matricon J, Meleine M, Gelot A, et al. Review article: associations between immune activation, intestinal permeability and the irritable bowel syndrome. Aliment Pharmacol Ther 2012;36:1009-1031.

18. Choi YJ, Hwang SW, Kim N, Park JH, Oh JC, Lee DH. Association between SLC6A4 serotonin transporter gene lainked polymorphic region and ADRA2A -1291C $>\mathrm{G}$ and irritable bowel syndrome in Korea. J Neurogastroenterol Motil 2014;20:388-399.

19. Choi YJ, Kim N, Yoon H, et al. Overlap between irritable bowel syndrome and functional dyspepsia including subtype analyses. J Gastroenterol Hepatol 2017;32:1553-1561.

20. Kim JJ, Kim N, Choi YJ, Kim JS, Jung HC. Increased TRPV1 and PAR2 mRNA expression levels are associated only with the esophageal reflux symptoms, but not with the extraesophageal reflux symptoms. Medicine (Baltimore) 2016;95:e4387.

21. Kim JJ, Kim N, Park JH, et al. Comparison of tight junction protein- 
related gene mRNA expression levels between male and female gastroesophageal reflux disease patients. Gut Liver 2018;12:411-419.

22. Song CH, Kim N, Sohn SH, et al. Effects of 17beta-estradiol on colonic permeability and inflammation in an azoxymethane/dextran sulfate sodium-induced colitis mouse model. Gut Liver 2018;12:682-693.

23. Ma J, Altomare A, Guarino M, et al. HCl-induced and ATP-dependent upregulation of TRPV1 receptor expression and cytokine production by human esophageal epithelial cells. Am J Physiol Gastrointest Liver Physiol 2012;303:G635-G645.

24. Yoshida N, Kuroda M, Suzuki T, et al. Role of nociceptors/neuropeptides in the pathogenesis of visceral hypersensitivity of nonerosive reflux disease. Dig Dis Sci 2013;58:2237-2243.

25. Son HJ, Sohn SH, Kim N, et al. Effect of estradiol in an azoxymethane/ dextran sulfate sodium-treated mouse model of colorectal cancer: implication for sex difference in colorectal cancer development. Cancer Res Treat 2019;51:632-648.

26. Choi YJ, Kim N, Kim J, Lee DH, Park JH, Jung HC. Upregulation of vanilloid receptor-1 in functional dyspepsia with or without Helicobacter pylori infection. Medicine (Baltimore) 2016;95:e3410.

27. Xu XJ, Zhang YL, Liu L, Pan L, Yao SK. Increased expression of nerve growth factor correlates with visceral hypersensitivity and impaired gut barrier function in diarrhoea-predominant irritable bowel syndrome: a preliminary explorative study. Aliment Pharmacol Ther 2017;45:100114.

28. Dothel G, Barbaro MR, Boudin H, et al. Nerve fiber outgrowth is increased in the intestinal mucosa of patients with irritable bowel syndrome. Gastroenterology 2015;148:1002-1011, e4.

29. Steinkamp M, Geerling I, Seufferlein T, et al. Glial-derived neurotrophic factor regulates apoptosis in colonic epithelial cells. Gastroenterology 2003;124:1748-1757.
30. Shieh KR, Yi CH, Liu TT, et al. Evidence for neurotrophic factors associating with TRPV1 gene expression in the inflamed human esophagus. Neurogastroenterol Motil 2010;22:971-977, e252.

31. Lee SH. Intestinal permeability regulation by tight junction: implication on inflammatory bowel diseases. Intest Res 2015;13:11-18.

32. Nusrat A, Turner JR, Madara JL. Molecular physiology and pathophysiology of tight junctions. IV. Regulation of tight junctions by extracellular stimuli: nutrients, cytokines, and immune cells. Am J Physiol Gastrointest Liver Physiol 2000;279:G851-G857.

33. Zeisel MB, Dhawan P, Baumert TF. Tight junction proteins in gastrointestinal and liver disease. Gut 2019;68:547-561.

34. Van Itallie CM, Anderson JM. Architecture of tight junctions and principles of molecular composition. Semin Cell Dev Biol 2014;36:157-165.

35. Piche T, Barbara G, Aubert P, et al. Impaired intestinal barrier integrity in the colon of patients with irritable bowel syndrome: involvement of soluble mediators. Gut 2009;58:196-201.

36. Dunlop SP, Jenkins D, Neal KR, Spiller RC. Relative importance of enterochromaffin cell hyperplasia, anxiety, and depression in postinfectious IBS. Gastroenterology 2003;125:1651-1659.

37. Gwee KA, Collins SM, Read NW, et al. Increased rectal mucosal expression of interleukin 1beta in recently acquired post-infectious irritable bowel syndrome. Gut 2003;52:523-526.

38. Dinan TG, Quigley EM, Ahmed SM, et al. Hypothalamic-pituitarygut axis dysregulation in irritable bowel syndrome: plasma cytokines as a potential biomarker? Gastroenterology 2006;130:304-311.

39. Liebregts T, Adam B, Bredack C, et al. Immune activation in patients with irritable bowel syndrome. Gastroenterology 2007;132:913-920.

40. Chang L, Adeyemo M, Karagiannides I, et al. Serum and colonic mucosal immune markers in irritable bowel syndrome. Am J Gastroenterol 2012;107:262-272. 